\title{
Improvement in Function after Lasmiditan Treatment: Post Hoc Analysis of Data from Phase 3 Studies
}

Timothy Smith · John H. Krege · Suchitrita S. Rathmann •

Sherie A. Dowsett - Ann Hake - Emel S. M. Nery • Brandy R. Matthews ·

Erin G. Doty

Received: February 21, 2020 / Published online: May 23, 2020

(C) The Author(s) 2020

\section{ABSTRACT}

Introduction: Migraine is associated with substantial functional impairment and affects many aspects of daily life.

Methods: Using data from SAMURAI and SPARTAN (double-blind, placebo-controlled, phase 3 studies) and GLADIATOR (an open-label, phase 3 study enrolling patients who had completed SAMURAI or SPARTAN), we assessed the effects of lasmiditan on migraine-related functional disability at multiple time points from 0.5 to $48 \mathrm{~h}$ post dose by asking patients to rate how much the migraine was interfering

Digital Features To view digital features for this article go to https://doi.org/10.6084/m9.figshare.12000105.

Electronic supplementary material The online version of this article (https://doi.org/10.1007/s40120020-00185-5) contains supplementary material, which is available to authorized users.

T. Smith

StudyMetrix Research, St. Peters, MO, USA

J. H. Krege - S. S. Rathmann - S. A. Dowsett - A. Hake

· E. S. M. Nery · B. R. Matthews · E. G. Doty ( $₫)$

Eli Lilly and Company, Indianapolis, IN, USA

e-mail: doty_erin_gautier@lilly.com

A. Hake - B. R. Matthews

Department of Neurology, Indiana University

School of Medicine, Indianapolis, IN, USA with normal activities. Pooled data from SAMURAI and SPARTAN (SAMURAI + SPARTAN) and data from GLADIATOR were analyzed using the intention-to-treat populations.

Results: For SPARTAN + SAMURAI, significantly more patients who received lasmiditan at any dose versus placebo reported freedom from migraine-related functional disability at every timepoint from $2 \mathrm{~h}$ post dose, and this difference persisted to $48 \mathrm{~h}(p<0.05)$. Significant differences from placebo in freedom from migraine-related functional disability commenced at $1 \mathrm{~h}$ post dose for lasmiditan $200 \mathrm{mg}$, $1.5 \mathrm{~h}$ for lasmiditan $100 \mathrm{mg}$, and $2 \mathrm{~h}$ for lasmiditan $50 \mathrm{mg}$. Findings from GLADIATOR supported those from SAMURAI + SPARTAN.

Conclusion: All doses of lasmiditan resulted in an improvement in migraine-related functional disability that persisted to $48 \mathrm{~h}$. In SAMURAI + SPARTAN, a significant difference from placebo was observed as early as $1 \mathrm{~h}$ post dose.

Trial registration at clinicaltrials.gov: SAMURAI (NCT02439320), SPARTAN (NCT02605174), and GLADIATOR (NCT02565186).

Keywords: Disability; Function; Lasmiditan; Migraine 


\section{Key Summary Points}

Why carry out this study?

Migraine is a neurological disease characterized by attacks of moderate-tosevere head pain associated with other symptoms. It has a substantial impact on the ability of the patient to function and affects many aspects of daily life, placing significant burdens on both the patient and their family.

Lasmiditan is a selective serotonin $5-\mathrm{HT}_{1 \mathrm{~F}}$ receptor agonist (a ditan) that was recently approved by the FDA for the acute treatment of migraine with and without aura in adults.

The aim of this post hoc analysis was to assess the effects of lasmiditan on migraine-related functional disability over time for up to $48 \mathrm{~h}$ post dose, using data from three phase 3 studies of lasmiditan.

What was learned from the study?

Lasmiditan treatment results in an improvement in migraine-related functional disability as early as $1 \mathrm{~h}$ post dose, and the effect persists for $48 \mathrm{~h}$.

Patients who report pain freedom with lasmiditan treatment are more likely to achieve freedom from functional disability than patients who do not report pain freedom at all time points from 0.5 to $48 \mathrm{~h}$ post dose.

Common adverse events associated with lasmiditan treatment do not generally appear to negatively impact migrainerelated functional disability.

\section{INTRODUCTION}

Migraine is a neurological disease characterized by attacks of moderate-to-severe head pain associated with other symptoms, including nausea, vomiting, photophobia, phonophobia, dizziness, and cognitive impairment [1]. It has a substantial impact on the patient's ability to function and affects many aspects of daily life [2-5], placing significant burdens on both the patient and their family. The Global Burden of Diseases, Injuries, and Risk Factors (GBD) Study (2016) found migraine to be the sixth most prevalent disease; it ranked second in terms of years of life lived with disability, and was among the ten most disabling disorders in each of the GBD regions [6].

Migraine attacks interfere with normal daily activities. Dodick et al. [7] recommended that an important outcome measure for acute treatment studies with real-world meaning for patients is the ability to participate in normal activities. Acute treatments would be expected to improve patient-reported ability to function in normal daily activities.

Lasmiditan is a selective serotonin $5-\mathrm{HT}_{1 \mathrm{~F}}$ receptor agonist (a ditan), recently approved by the FDA for the acute treatment of migraine with and without aura in adults. Two randomized double-blind placebo-controlled single-attack phase 3 trials, SAMURAI and SPARTAN, have been completed and the primary study findings published; in both studies, lasmiditan showed a benefit over placebo based on migraine-related functional disability at $2 \mathrm{~h}$ $[8,9]$. SAMURAI and SPARTAN were almost identical in design, so various analysis findings from the pooled dataset (SAMURAI + SPARTAN) have also been published [10-16]. Analysis of the SAMURAI + SPARTAN dataset using the modified intention-to-treat population (i.e., patients who had taken the study medication within $4 \mathrm{~h}$ of the onset of pain) showed that patients who took lasmiditan achieved freedom from migraine-related functional disability at $1 \mathrm{~h}$ post treatment for lasmiditan $200 \mathrm{mg}$, and at $1.5 \mathrm{~h}$ for lasmiditan $50 \mathrm{mg}$ and $100 \mathrm{mg}$ (based on significant differences in percentages versus placebo) [10]. These effects were maintained to 24 and $48 \mathrm{~h}$ [11]. Findings from the time points between $2 \mathrm{~h}$ and $24 \mathrm{~h}$ have not been previously published; nor has the time course for achieving freedom from migraine-related functional disability been explored in detail. 
Completers of SAMURAI or SPARTAN could participate in GLADIATOR, an open-label, phase 3 study to evaluate the safety and efficacy of lasmiditan for the intermittent, acute treatment of migraine attacks for up to 1 year [17].

The aim of this post hoc analysis was to assess the effects of lasmiditan on migraine-related functional disability over time for up to $48 \mathrm{~h}$ post dose using data from both SAMURAI + SPARTAN and from GLADIATOR. The effects of pain freedom and adverse events (AEs) on migraine-related functional disability were also explored.

\section{METHODS}

\section{Study Designs}

SAMURAI [8] and SPARTAN [9] were prospective, randomized, double-blind, multicenter, single-attack, phase 3 studies in which patients with migraine were randomized to lasmiditan $50 \mathrm{mg}$ (SPARTAN only), lasmiditan $100 \mathrm{mg}$, lasmiditan $200 \mathrm{mg}$, or placebo. Patient inclusion criteria were almost identical, except that SAMURAI (but not SPARTAN) excluded individuals with known coronary artery disease, clinically significant arrhythmia, and uncontrolled hypertension. Key inclusion criteria were a Migraine Disability Assessment (MIDAS) score of $\geq 11$ (moderate or severe migraine-associated disability), a history of migraine of $\geq 1$ year, $3-8$ migraine attacks per month with less than 15 headache days per month, and migraine onset before 50 years of age.

GLADIATOR [17] was a prospective, randomized, open-label, multicenter, phase 3 study enrolling patients who had completed either SAMURAI or SPARTAN. The study was designed to evaluate the safety (primary) and efficacy (secondary) of lasmiditan for the intermittent, acute treatment of migraine attacks for up to 1 year. In GLADIATOR, patients were re-randomized $1: 1$ to lasmiditan $100 \mathrm{mg}$ or $200 \mathrm{mg}$ and were asked to use lasmiditan as the first treatment for each new migraine attack of at least moderate severity.

In SAMURAI, SPARTAN, and GLADIATOR, a second dose of the study drug could be taken for rescue or recurrence, and was to be taken $2-24 \mathrm{~h}$ after the first dose. In SAMURAI and SPARTAN, for the second dose, patients originally assigned lasmiditan were randomized to the same active dose or placebo (2:1 ratio), and patients originally assigned placebo received placebo. In GLADIATOR, for the second dose, patients were randomized to the same dose of lasmiditan (100 $\mathrm{mg}$ or $200 \mathrm{mg}$ ) as they received for the first dose.

SAMURAI, SPARTAN, and GLADIATOR conformed with the Helsinki Declaration of 1964, as revised in 2013, concerning human and animal rights. The study protocols were approved by an independent ethics committee or institutional review board at each study site (see the Electronic supplementary material, ESM, for details). All patients provided written informed consent for study participation prior to the start of the study. Springer's policy concerning informed consent was followed.

\section{Efficacy Endpoints}

Migraine-related functional disability, an exploratory efficacy endpoint in all studies, was assessed at multiple time points by asking patients, "How much is your migraine interfering with your normal activities?" This had four response options: "not at all," "mild interference," "marked interference," or "need complete bed rest." Freedom from migraine-related functional disability was defined as having disability "not at all" at a given time point post dose. An electronic diary was used to collect these data at $0.5,1,1.5,2,3,4,24$, and $48 \mathrm{~h}$ post dose in SAMURAI and SPARTAN, and at $0.5,1$, $2,4,24$, and $48 \mathrm{~h}$ post dose in GLADIATOR.

Head pain severity was assessed at these same time points, via the electronic diary, using a headache severity rating scale. Pain freedom at $2 \mathrm{~h}$ was a primary endpoint of both SAMURAI and SPARTAN.

At $2 \mathrm{~h}$, patients were asked the Patient Global Impression of Change (PGIC) question [18], "How do you feel after taking study medication?" Responses were captured using a 7-point Likert scale from "very much better" to "very much worse." The PGIC is an integrated 
measure of drug tolerability and efficacy, measuring the patient's view of improvement or decline in clinical status after study drug exposure.

\section{Safety Endpoints}

At the same timepoints specified for efficacy endpoints, the electronic diary asked whether the patient felt anything unusual since taking the study medication that they had not felt with a migraine previously. When a patient answered "yes," they were contacted by the study site to determine if an $\mathrm{AE}$ had been experienced. AEs that occurred or worsened within $48 \mathrm{~h}$ of dosing were considered treatment emergent. Common treatment-emergent adverse events (TEAEs) were defined as those with $\geq 2 \%$ incidence in any lasmiditan dose group.

\section{Statistical Analyses}

Data from the SAMURAI and SPARTAN studies were pooled (SAMURAI + SPARTAN) for the analyses. For GLADIATOR, first-attack data were used. SAMURAI + SPARTAN and GLADIATOR data were analyzed separately, and analyses were post hoc unless otherwise specified.

To assess the effect of lasmiditan on migraine-related functional disability, we determined the percentage of patients reporting no disability by treatment group at each available timepoint. Data were analyzed using the intention-to-treat (ITT) population, defined as all randomized patients who used at least one dose of study drug and had any post-dose headache severity or symptom assessments.

In the lasmiditan phase 3 studies, a second dose of study drug or other approved medication could be taken 2-24 h after the first dose if needed for rescue or recurrence. This resulted in missing data after the 2-h timepoint, as the electronic diary clock reset for timepoints post second dose. To account for these missing data beyond $2 \mathrm{~h}$, we employed two different data analysis methods: a nonresponder imputation (NRI) approach and an observed case analysis. Using the NRI method, denominators for calculating migraine-related functional disability percentages were the total number of patients in the ITT population in each treatment group. At any timepoint, if responses were not available, they were considered missing. For patients who took a second dose of the study drug or other approved rescue medication, data after the 2-h timepoint were also considered missing. For statistical testing, missing data were included in the "any interference" grouping. The NRI approach potentially underestimates treatment efficacy by treating missing data as treatment failures (patients were considered not to be disability free when they could have been). For the observed case analysis, denominators for calculating migraine-related functional disability percentages were the total patient counts in each treatment group at the specific time point, so that missing data were excluded from the analysis. For patients who took a second dose of study drug or approved rescue medication, data after the $2-\mathrm{h}$ timepoint were also excluded. The observed case approach potentially overestimates treatment efficacy by analyzing only available data at the timepoint. For SAMURAI + SPARTAN, the Cochran-Mantel-Haenszel test with stratification by study was used to compare the percentage with no interference in the lasmiditan dose groups to placebo at each timepoint.

To determine whether there was an association between pain freedom and migraine-related functional disability, the percentages of patients with disability 'not at all' for those who were pain free and those who were not pain free were summarized by treatment group at each timepoint, and the association between pain freedom and migraine-related functional disability was tested within each treatment group using Cochran-Mantel-Haenszel tests with stratification by study. Since it is not always clear whether a patient's response to the migraine-related disability question is based on the interference with their activities caused by migraine, caused by TEAEs, or caused by both, we assessed whether there was any association between pain freedom and the response to the PGIC question ("How do you feel after taking study medication?"); we summarized the percentages of patients who gave a PGIC response of "much better" or "very much better" and were pain free or not pain free at $2 \mathrm{~h}$ by 
treatment group, and tested the association between pain freedom and PGIC response of "much better" or "very much better." In both cases, an observed case analysis was performed.

To determine whether there was any association between reporting of individual common TEAEs and migraine-related functional disability, we compared the percentages of patients with disability "not at all" at each timepoint between those who reported a common TEAE up to that timepoint and those who did not; TEAEs that had resolved before a specific timepoint were not excluded from that timepoint.

For the pain freedom/migraine-related functional disability, the pain freedom/PGIC response and TEAE/migraine-related functional disability analyses for SAMURAI + SPARTAN data from only the lasmiditan dose groups were included (i.e., placebo data were excluded).

\section{RESULTS}

\section{Migraine-Related Functional Disability over Time}

In SAMURAI + SPARTAN, using the NRI method, significant differences from placebo $(p<0.05)$ in freedom from migraine-related functional disability commenced at $1 \mathrm{~h}$ post dose for lasmiditan $200 \mathrm{mg}, 1.5 \mathrm{~h}$ for lasmiditan $100 \mathrm{mg}$, and $2 \mathrm{~h}$ for lasmiditan $50 \mathrm{mg}$; these differences persisted to $48 \mathrm{~h}$ (Fig. 1a). The observed case analysis showed a similar pattern over time, but statistical differences between active treatment and placebo were observed only at timepoints up to $4 \mathrm{~h}$ post dose (Fig. 1b). In GLADIATOR (first-attack data), the percentage of patients free from migraine-related functional disability increased over time from 0.5 to $4 \mathrm{~h}$ post dose (NRI method) and from 0.5 to $48 \mathrm{~h}$ post dose (observed case analysis) (Fig. 2a and b). For all dose groups in both SAMURAI + SPARTAN and GLADIATOR, the percentage of patients reporting no interference increased over time from $0.5 \mathrm{~h}$ post dose, while the percentages reporting marked interference or a need for complete bed rest decreased. The percentages reporting mild interference decreased from $1.5 \mathrm{~h}$ post dose (Figs. 1 and 2).

\section{Pain Freedom and Migraine-Related Functional Disability over Time}

In both SAMURAI + SPARTAN (lasmiditan treatment groups only) and GLADIATOR, a significantly greater percentage of patients in the pain-free group versus not pain-free group reported no migraine-related disability at all time points post dose $(p<0.001$ in all cases) (Fig. 3a, b). Overall, 62-98\% (depending on the dose group, post-dose timepoint, and study) who were pain free reported no migraine-related disability. Those pain-free patients who were not disability free generally reported only mild interference with daily activity (data not shown). In the pain-free group at timepoints up to $3 \mathrm{~h}$, there was some evidence of a dose effect; the percentage of patients who had no disability decreased with increasing lasmiditan dose.

\section{Pain Freedom and PGIC at $2 \mathrm{~h}$}

In both SAMURAI + SPARTAN and GLADIATOR, a significantly greater percentage of patients in the pain-free group versus not pain-free group reported feeling 'much better' or 'very much better' 2 hours post dose ( $p<0.001$ in all cases) (Fig. 4a, b). Overall, 84-92\% of patients (depending upon the dose group and study) who were pain free reported feeling "much better" or "very much better" $2 \mathrm{~h}$ post dose.

\section{Association Between Common Adverse Event Occurrence and Migraine-Related Functional Disability over Time}

In SAMURAI + SPARTAN (pooled lasmiditan dose groups), patients who experienced dizziness, fatigue, somnolence, or paresthesia did not generally exhibit greater disability than patients who did not experience a TEAE at any timepoint (Fig. 5). In GLADIATOR, the number of cases of each common TEAE was low; findings were generally similar to those in SAMURAI + SPARTAN, although more variable (data not shown). 


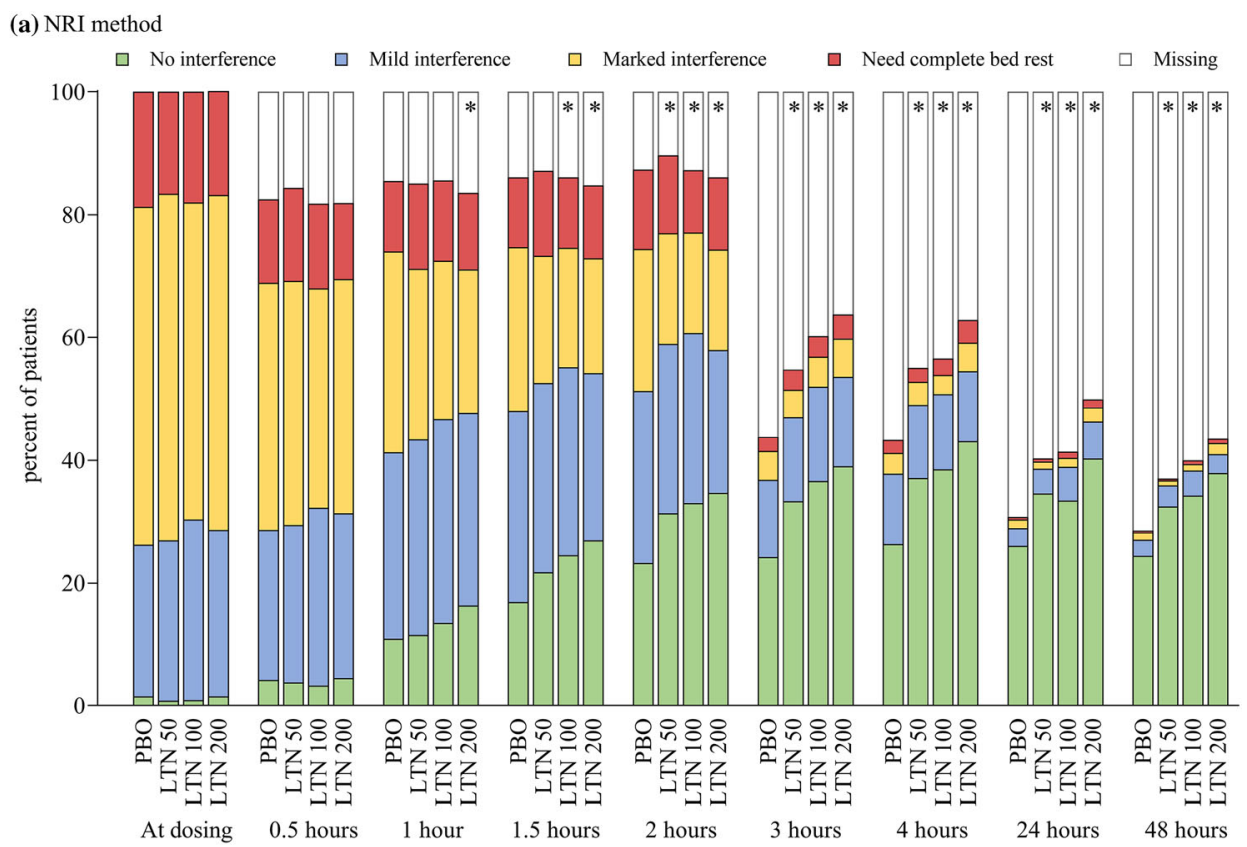

Treatment groups: PBO, N=1130; LTN 50, N=598; LTN 100, N=1133; LTN 200, N=1120

(b) Observed case analysis



${ }^{\mathrm{a} N u m b e r}$ of patients for each treatment group at each time point

${ }^{*} \mathrm{p}<0.05 \mathrm{v}$ PBO for percent of patients with no interference with normal activity Abbreviations: LTN, lasmiditan; PBO, placebo

Fig. 1 Migraine-related functional disability by treatment group and timepoint - SAMURAI+SPARTAN by a NRI method and $\mathbf{b}$ observed case analysis 
(a) NRI method

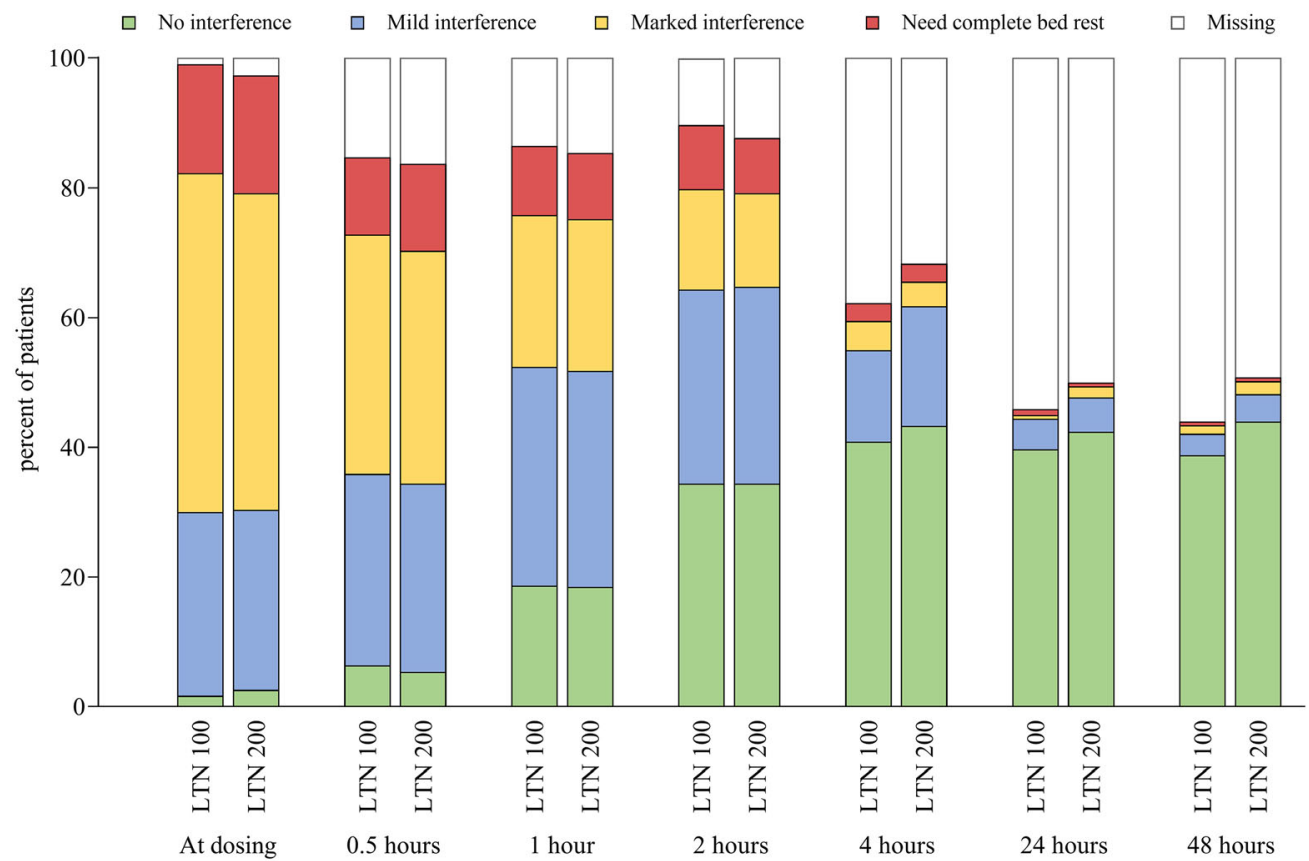

Treatment groups: LTN 100, N=941; LTN 200, N=990

(b) Observed case analysis

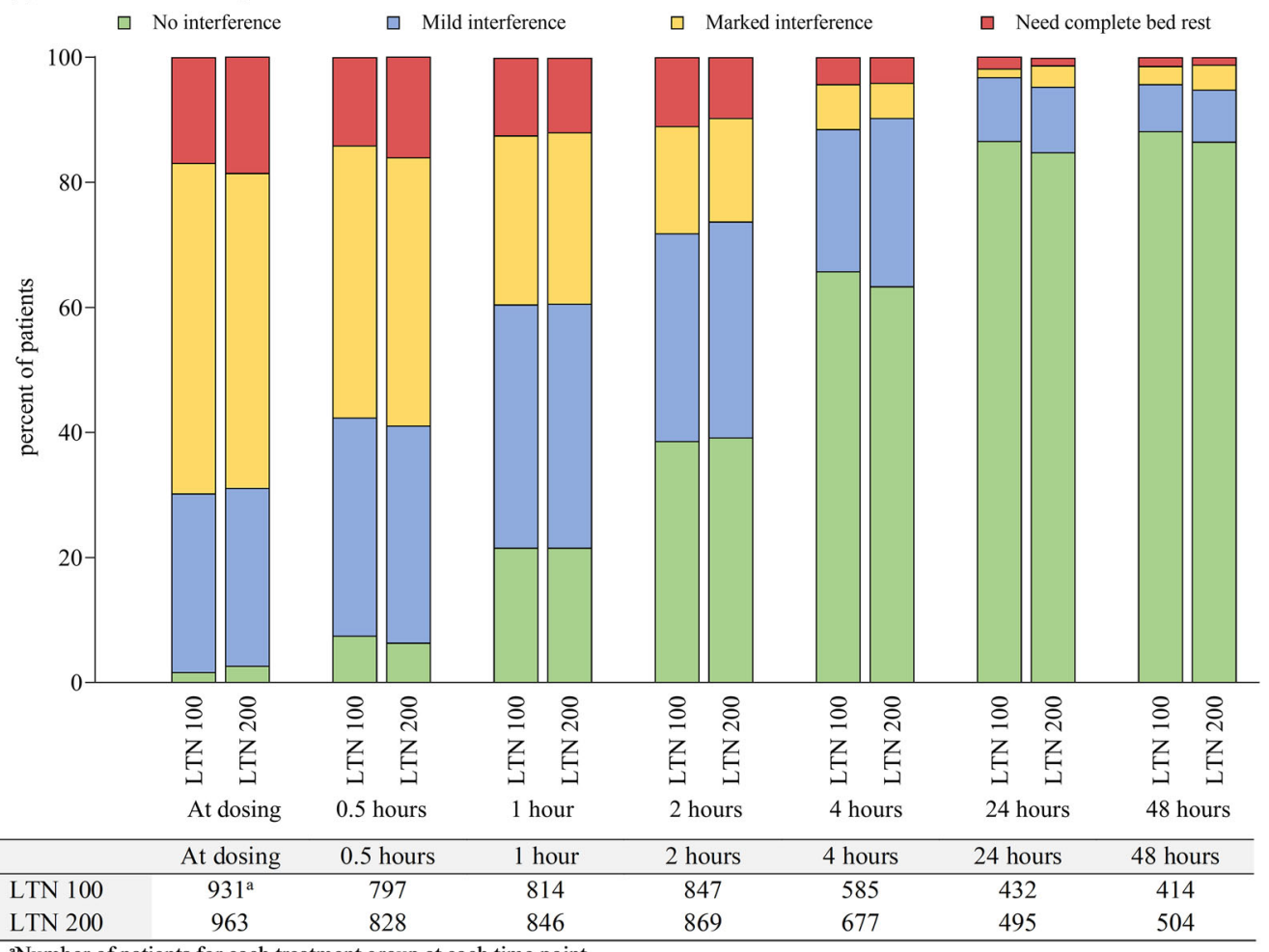

aNumber of patients for each treatment group at each time point

Abbreviations: LTN, lasmiditan

Fig. 2 Migraine-related functional disability by treatment group and time point - GLADIATOR (first attack) by a NRI method and $\mathbf{b}$ observed case analysis 
(a) SAMURAI+SPARTAN

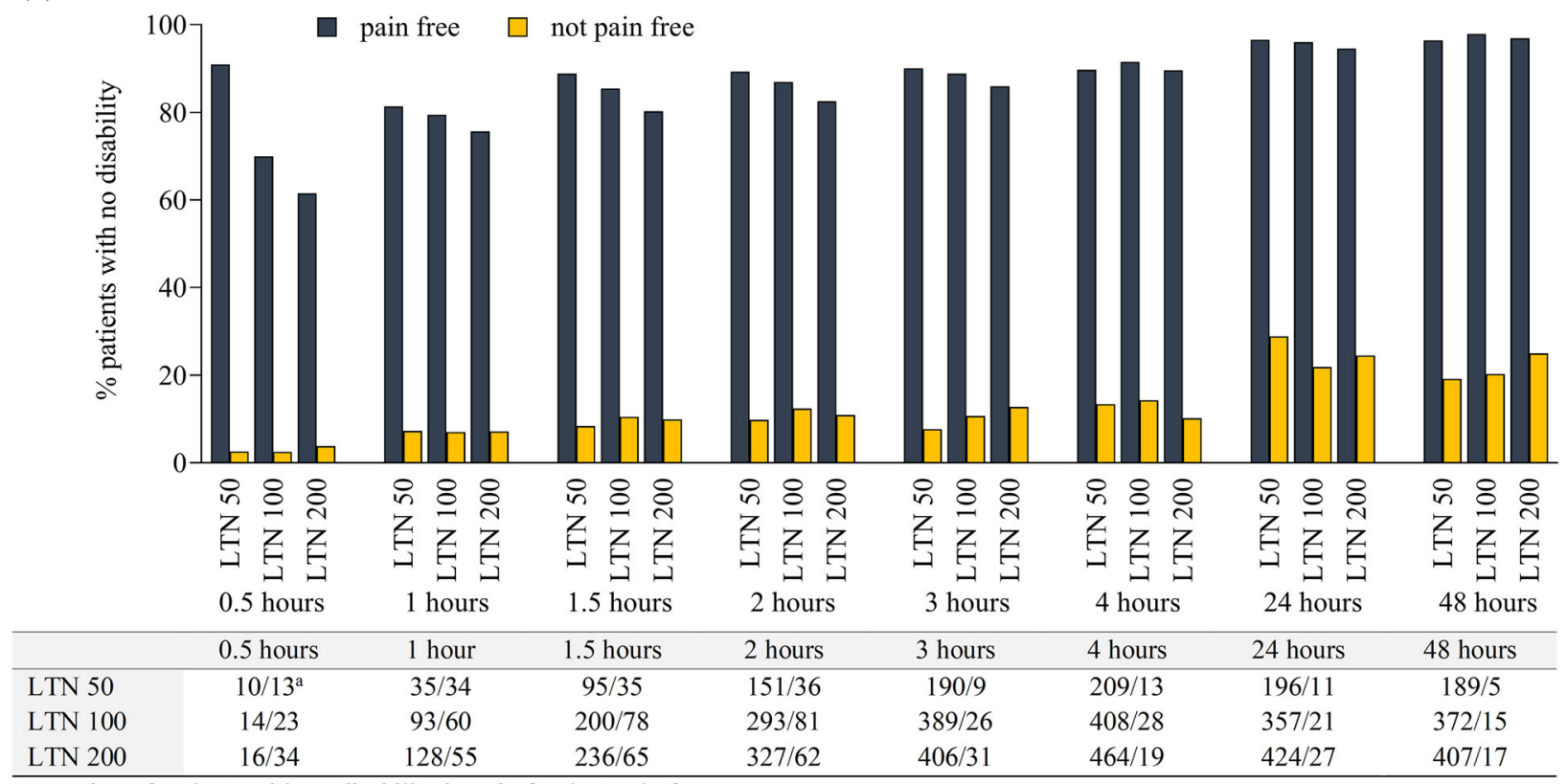

${ }^{a}$ Number of patients with no disability in pain free/not pain free group

(b) GLADIATOR

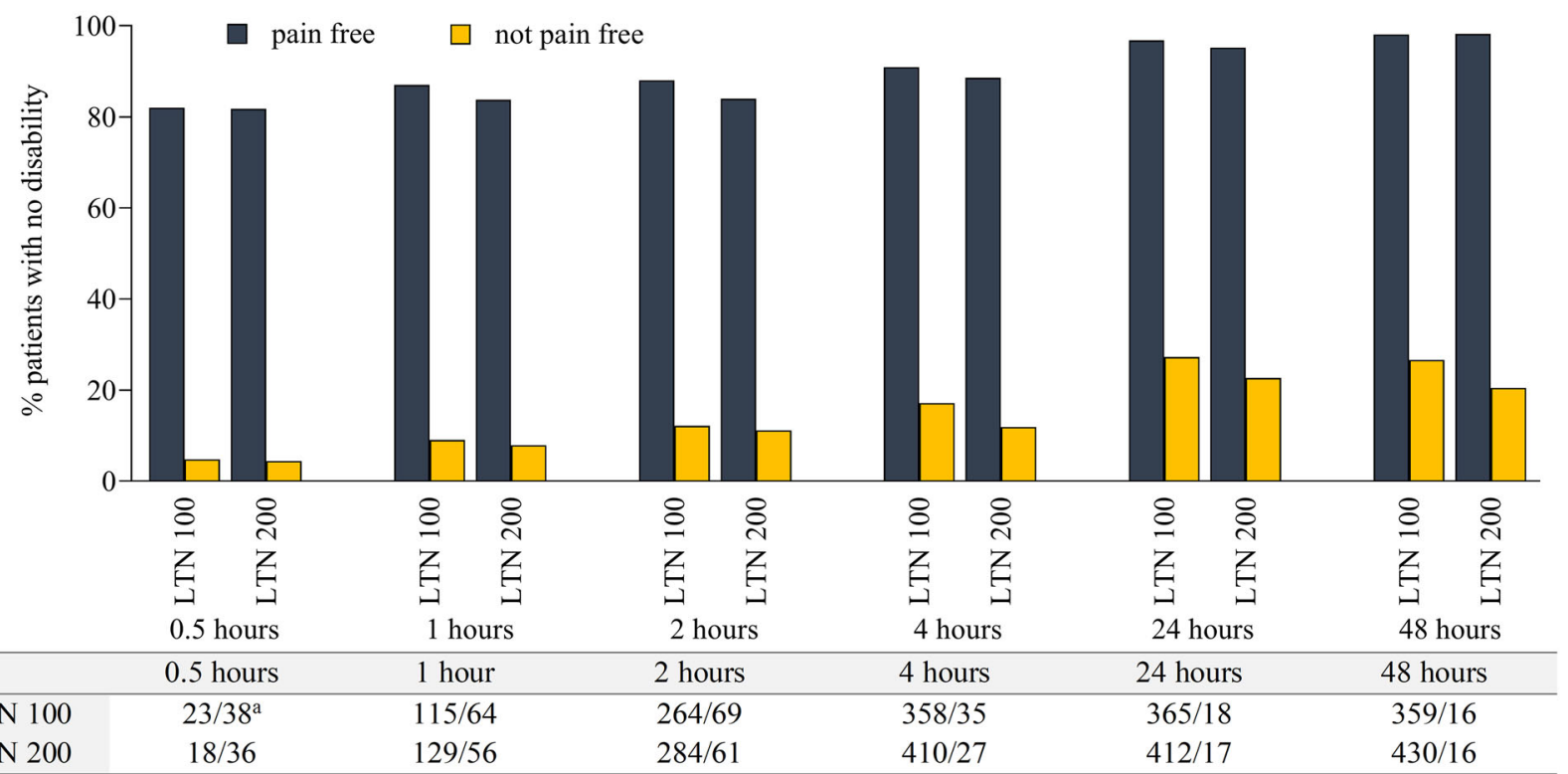

${ }^{\mathrm{a} N u m b e r}$ of patients with no disability in pain free/not pain free group

$\mathrm{p}<0.001$ for percent of patients with no disability in pain free vs not pain free group at all time points

Abbreviations: LTN, lasmiditan

Fig. 3 Percent of patients with no disability by pain freedom (yes versus no) over time in a SAMURAI+SPARTAN and $\mathbf{b}$ GLADIATOR (observed case analysis) 
(a) SAMURAI+SPARTAN

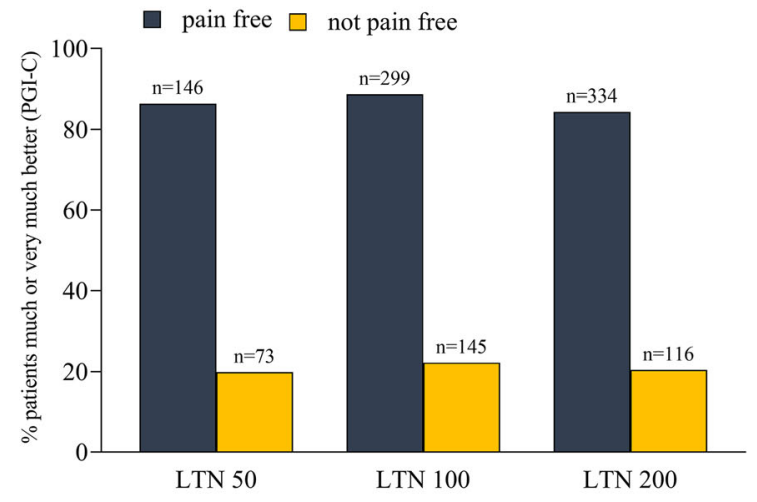

(b) GLADIATOR

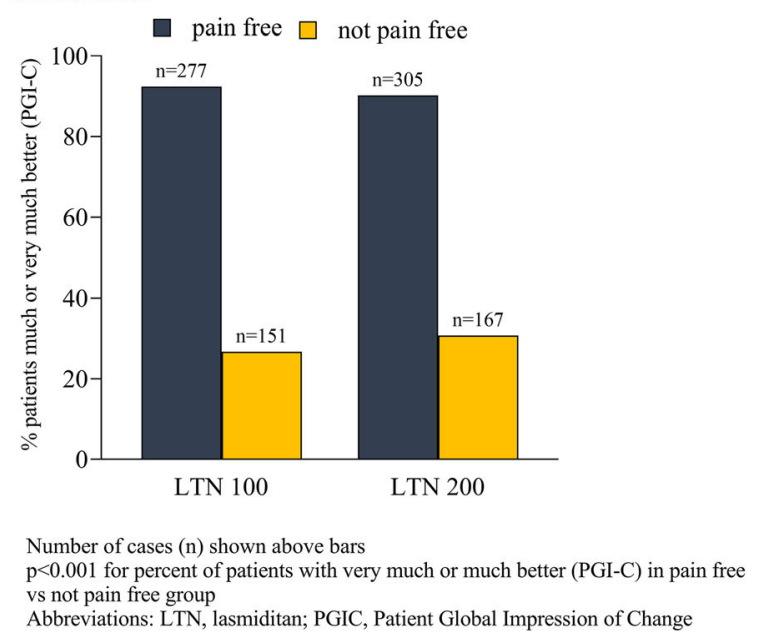

Fig. 4 Percent of patients who were much or very much better on the Global Impression of Change by pain freedom (yes versus no) at 2 hours in a SAMURAI+SPARTAN and b GLADIATOR (observed case analysis)

\section{DISCUSSION}

In this post hoc analysis of data from phase 3 studies, lasmiditan use was associated with an improvement in migraine-related functional disability. In SAMURAI + SPARTAN, based on findings from the NRI analysis, lasmiditan resulted in a significant improvement in migraine-related functional disability compared with placebo, commencing at $1 \mathrm{~h}$ post dose for lasmiditan $200 \mathrm{mg}, 1.5 \mathrm{~h}$ for lasmiditan $100 \mathrm{mg}$, and $2 \mathrm{~h}$ for lasmiditan $50 \mathrm{mg}$, and persisting to $48 \mathrm{~h}$ for all doses. For SAMURAI + SPARTAN, the observed case analysis showed a similar pattern over time, but statistical differences between the active treatment and placebo were seen only at timepoints up to $4 \mathrm{~h}$ post dose. For the observed case analysis, data from patients who took a second dose of the study drug for rescue or recurrence (i.e., patients who were potentially more difficult to treat) were excluded; this was more frequent in the placebo group. Excluding these data presumably leaves a sample of patients with less severe migraine, and reduces the ability to see an effect of the active drug. Findings from GLADIATOR were consistent with those from SAMURAI + SPARTAN, with an improvement in migrainerelated functional disability over the $48 \mathrm{~h}$. While only first-attack data are presented, the findings were consistent across subsequent attacks in GLADIATOR (unpublished data).

In both SAMURAI + SPARTAN and GLADIATOR, a clear relationship between being pain free and reporting no migraine-related 

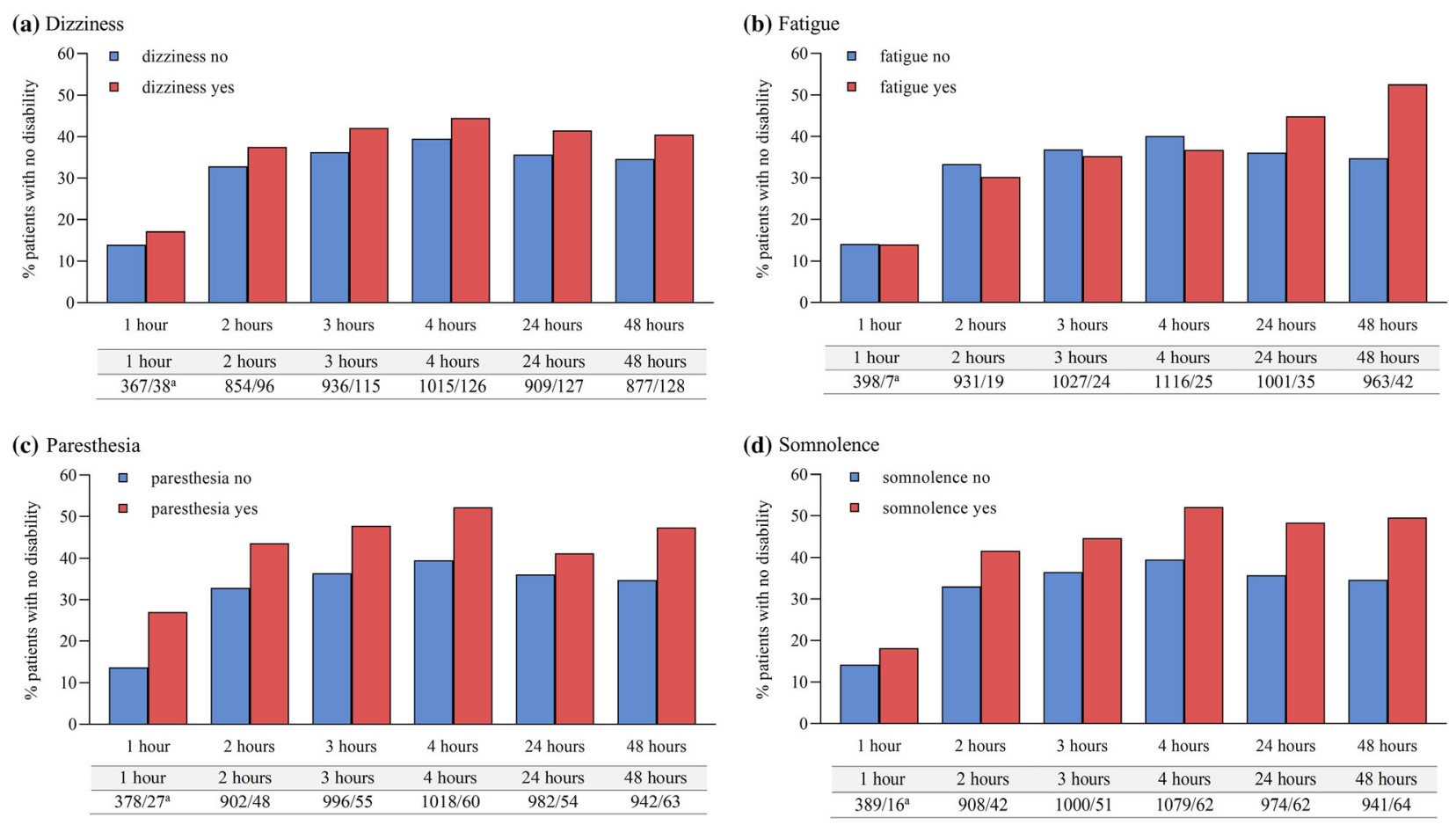

${ }^{\mathrm{a}}$ Number of patients with no disability where common TEAE not reported/reported

Fig. 5 Percent of patients with no disability by reporting (yes versus no) of common TEAE - SAMURAI+SPARTAN (lasmiditan dosing groups only)

disability was evident at all time points post dose. At earlier time points (up to $3 \mathrm{~h}$ ) in SAMURAI + SPARTAN, there appeared to be a dose response in the pain-free group, with fewer patients reporting being disability free with increasing lasmiditan dose. For those who did experience disability in this context, it was generally only mild interference with daily activity. This lasmiditan dose response suggests that the occurrence of TEAEs may have influenced patient response to the disability question. A common concern expressed regarding the interpretation of the disability question ("How much is your migraine interfering with your normal activities?") is that it is unclear if a patient's response is based on the interference with their activities caused by migraine, caused by TEAEs, or caused by both. However, the effects of common TEAEs associated with lasmiditan use on migraine-related function generally appear to be minimal or even potentially positive (see the discussion of TEAEs below).
Due to some of the challenges involved in interpreting the answer to the migraine-related disability question, we also assessed the association between pain status at $2 \mathrm{~h}$ and how a patient responded to the PGIC question "How do you feel after taking study medication?" This question seeks to evaluate the overall effect of treatment on patient well-being, taking into account both the benefits of reducing migraine symptoms and any adverse effects of treatment. In SAMURAI + SPARTAN and GLADIATOR, feeling much or very much better was clearly associated with being pain free.

While no formal comparisons were made, the percentage of patients who reported no disability was generally higher for patients who experienced a common TEAE than for patients who did not experience a common TEAE. The only exception was for fatigue at earlier timepoints. This relationship between the occurrence of common TEAEs and disability has been previously reported for the 2-h post-dose 
timepoint $[14,15]$. Based on the findings of the current analysis, the pattern was generally maintained across the $48 \mathrm{~h}$; fatigue was the only exception, with a less consistent pattern across the $48 \mathrm{~h}$.

There are limitations of this study. The majority of analyses were post hoc. Mainly as a result of excluding data for patients who took a second dose, sample sizes were reduced after $2 \mathrm{~h}$. Also, our measure of migraine-related functional disability has not been formally validated, although this measure has been used in other migraine acute treatment trials [19-22]. The authors have not been able to identify any other validated patient-reported outcome measure of in-the-moment function at timepoints earlier than $24 \mathrm{~h}$.

\section{CONCLUSIONS}

All doses of lasmiditan resulted in a significant improvement in migraine-related functional disability, which commenced as early as $1 \mathrm{~h}$ post dose and persisted to $48 \mathrm{~h}$. Freedom from migraine-related functional disability as well as improvement in overall clinical status (as measured using the PGIC) were associated with pain freedom. Common adverse events associated with lasmiditan treatment did not generally appear to negatively impact migraine-related functional disability.

\section{ACKNOWLEDGEMENTS}

Funding. The authors disclosed receipt of the following financial support for the research, and publication of this article: Payment of journal's Rapid Service Fees; The SAMURAI, SPARTAN, and GLADIATOR studies were sponsored by CoLucid Pharmaceuticals, a wholly owned subsidiary of Eli Lilly and Company.

Medical Writing, Editorial, and Other Assistance. The scientific writer (SAD) made a significant contribution to this manuscript, fulfilled authorship requirements, and is listed as an author.
Authorship. All named authors meet the International Committee of Medical Journal Editors (ICMJE) criteria for authorship for this article, take responsibility for the integrity of the work as a whole, and have given their approval for this version to be published.

Disclosures. Timothy Smith reports financial relationships with Alder-Lundbeck, Allergan, Amgen, Biohaven, Charleston Labs, Electrocore, Impel, Eli Lilly and Company, Novartis Novo Nordisk, Satsuma, Theranica, United Health Group, and Vorso Technologies; he received no payment for manuscript preparation. John H. Krege, Suchitrita S. Rathmann, Sherie A. Dowsett, Ann Hake, Emel S.M. Nery, Brandy R. Matthews, and Erin G. Doty are fulltime employees and minor stockholders at Eli Lilly and Company.

Compliance with Ethics Guidelines. SAMURAI, SPARTAN, and GLADIATOR conformed with the Helsinki Declaration of 1964, as revised in 2013, concerning human and animal rights. The study protocols were approved by an independent ethics committee or institutional review board at each study site (see the ESM for details). All patients provided written informed consent for study participation prior to the start of the study. Springer's policy concerning informed consent has been followed.

Data Availability. Eli Lilly and Company provides access to all individual participant data collected during the trial, after anonymization, with the exception of pharmacokinetic or genetic data. Data are available to request 6 months after the indication studied has been approved in the US and EU and after primary publication acceptance, whichever is later. No expiration date of data requests is currently set once data are made available. Access is provided after a proposal has been approved by an independent review committee identified for this purpose and after receipt of a signed data sharing agreement. Data and documents, including the study protocol, statistical analysis plan, clinical study report, and blank or annotated case report forms, will be provided in a secure 
data sharing environment. For details on submitting a request, see the instructions provided at http://www.vivli.org.

Open Access. This article is licensed under a Creative Commons Attribution-NonCommercial 4.0 International License, which permits any non-commercial use, sharing, adaptation, distribution and reproduction in any medium or format, as long as you give appropriate credit to the original author(s) and the source, provide a link to the Creative Commons licence, and indicate if changes were made. The images or other third party material in this article are included in the article's Creative Commons licence, unless indicated otherwise in a credit line to the material. If material is not included in the article's Creative Commons licence and your intended use is not permitted by statutory regulation or exceeds the permitted use, you will need to obtain permission directly from the copyright holder. To view a copy of this licence, visit http://creativecommons.org/licenses/by$\mathrm{nc} / 4.0 /$

\section{REFERENCES}

1. Dodick DW. Migraine. Lancet. 2018;391:1315-30.

2. Abu Bakar N, Tanprawate S, Lambru G, et al. Quality of life in primary headache disorders: a review. Cephalalgia. 2016;36:67-91.

3. Leonardi M, Raggi A, Ajovalasit D, et al. Functioning and disability in migraine. Disabil Rehabil. 2010;32:S23-32.

4. Chaushev N, Milanov I. Impact of migraine and migraine treatment on patient's capacity to work and quality of life. J Clin Med. 2009;2:26-31.

5. Lipton RB, Bigal ME, Diamond $\mathrm{M}$, et al. Migraine prevalence, disease burden, and the need for preventive therapy. Neurology. 2007;68:343-9.

6. Stovner LJ, Nichols E, Steiner TJ, et al. Global, regional, and national burden of migraine and tension-type headache, 1990-2016: a systematic analysis for the Global Burden of Disease Study 2016. Lancet Neurol. 2018;17:954-76.

7. Dodick DW, Turkel CC, DeGryse RE, et al. Assessing clinically meaningful treatment effects in controlled trials: chronic migraine as an example. J Pain. 2015;16:164-75.

8. Kuca B, Silberstein SD, Wietecha L, et al. Lasmiditan is an effective acute treatment for migraine: a phase 3 randomized study. Neurology. 2018;91:e2222-32.

9. Goadsby PJWL, Dennehy EB, Kuca B, Case MG, Aurora SK, Gaul C. Phase 3 randomized, placebocontrolled, double-blind study of lasmiditan for acute treatment of migraine. Brain. 2019;142: 1894-904.

10. Ashina M, Vasudeva R, Jin L, et al. Onset of efficacy following oral treatment with lasmiditan for the acute treatment of migraine: integrated results from 2 randomized double-blind placebo-controlled Phase 3 clinical studies. Headache. 2019;59: 1788-801.

11. Doty EG, Krege JH, Jin L, et al. Sustained responses to lasmiditan: results from post hoc analyses of two Phase 3 randomized clinical trials for acute treatment of migraine. Cephalalgia. 2019;39:1569-76.

12. Loo LS, Plato BM, Turner IM, et al. Effect of a rescue or recurrence dose of lasmiditan on efficacy and safety in the acute treatment of migraine: findings from the phase 3 trials (SAMURAI and SPARTAN). BMC Neur. 2019;19:191.

13. Loo LS, Ailani J, Schim J, et al. Efficacy and safety of lasmiditan in patients using concomitant migraine preventive medications: findings from SAMURAI and SPARTAN, two randomized phase 3 trials. J Headache Pain. 2019;20:84.

14. Tepper SJ, Krege JH, Lombard L, et al. Characterization of dizziness after lasmiditan usage: findings from the SAMURAI and SPARTAN acute migraine treatment randomized trials. Headache. 2019;59: $1052-62$.

15. Krege JH, Rizzoli PB, Liffick E, et al. Safety findings from Phase 3 lasmiditan studies for acute treatment of migraine: results from SAMURAI and SPARTAN. Cephalalgia. 2019;39:957-66.

16. Shapiro RE, Hochstetler HM, Dennehy EB, et al. Lasmiditan for acute treatment of migraine in patients with cardiovascular risk factors: post hoc analysis of pooled results from 2 randomized, double-blind, placebo-controlled, phase 3 trials. J Headache Pain. 2019;20:90.

17. Brandes JL, Klise S, Krege JH, et al. Interim results of a prospective, randomized, open-label, Phase 3 study of the long-term safety and efficacy of lasmiditan for acute treatment of migraine (the GLADIATOR study). Cephalalgia. 2019: 0333102419864132. 
18. Guy W, editor. Clinical global impressions. In: ECDEU assessment manual for psychopharmacology—revised. Rockville: US Department of Health, Education, and Welfare; 1976. p. 218-222.

19. Croop R, Goadsby PJ, Stock DA, et al. Efficacy, safety, and tolerability of rimegepant orally disintegrating tablet for the acute treatment of migraine: a randomised, phase 3, double-blind, placebo-controlled trial. Lancet (London, England). 2019;394: 737-45.

20. Lipton RB, Croop R, Stock EG, et al. Rimegepant, an oral calcitonin gene-related peptide receptor antagonist, for migraine. N Engl J Med. 2019;381: 142-9.

21. Dodick DW, Lipton RB, Ailani J, et al. Ubrogepant for the treatment of migraine. $\mathrm{N}$ Engl J Med. 2019;381:2230-41.

22. Lipton RB, Dodick DW, Ailani J, et al. Effect of ubrogepant vs placebo on pain and the most bothersome associated symptom in the acute treatment of migraine: the ACHIEVE II randomized clinical trial. JAMA. 2019;322:1887-98. 